\title{
Molecular cloning and characterization of a soybean GmMBY184 induced by abiotic stresses
}

\author{
Eunsook Chung $\cdot$ Koungmee Kim - Jai-Heon Lee
}

Received: 25 July 2012 / Accepted: 9 August 2012

(c) Korean Society for Plant Biotechnology

\begin{abstract}
Drought and high salinity stresses often imposes adverse effects on crop yield. MYB transcription factors have been shown to be an important regulator in defense responses to these environmental stresses. In this study, we have cloned and characterized a soybean gene GmMYB184 (Glycine max MYB transcription factor 184). Deduced amino acid sequences of GmMYB184 show highest homology with that from Vitis vinifera legume plant (75\%). Different expression patterns of GmMYB184 mRNA were observed subjected to drought, cold, high salinity stress and abscisic acid treatment, suggesting its role in the signaling events in the osmotic stress-related defense response. Subcellular localization studies demonstrated that the GFP-GmMYB184 fusion protein was localized in the nucleus. Using the yeast assay system, the C-terminal region of GmMYB184 was found to be essential for the transactivation activity. These results indicate that the GmMYB184 may play a role in abiotic stress tolerance in plant.
\end{abstract}

\section{Introduction}

As higher plants are sessile, they are directly exposed to various environmental stresses during their entire life cycle. Drought, high salinity, and extreme temperatures are the common abiotic stresses that impair the growth and development of plants. The plant tolerance to abiotic stress is mediated by signal transduction cascade and regulations of gene expression (Nakashima et al. 2009; Shinozaki and Yamaguchi-Shinozaki 2007). Transcription factors (TFs) act

*These authors contributed equally to this work.

E. S. Chung $\cdot$ K. M. Kim · J.-H. Lee $(\bowtie)$

BK21 Center for Silver-Bio Industrialization, College of Natural Resources and Life Science, Dong-A University, Busan 604-714, Korea

e-mail: jhnlee@dau.ac.kr at the upstream position of the signal transduction and gene regulatory network, which may amplify expression of a broad range of downstream genes in response to external signals. To date, several TFs such as MYB, ERF, bZIP and WRKY, have been implicated in the regulation of stress responses (Schwechheimer et al. 1998; Singh et al. 2002).

$M Y B$ gene was first identified from the 'oncogene' $v-M y b$ derived from the avian myeloblastosis virus (Klempnauer et al. 1982). Other MYB-related proteins such as $c-M y b$, $A-M y b$ and $B-M y b$ have been found in many vertebrates and other similar genes have been identified in insects, plants, fungi and slime moulds (Lipsick 1996). The MYB TFs are essential for the control of proliferation and differentiation in a number of cell types, and share the conserved MYB DNA-binding domain (DBD). This domain generally comprises up to three imperfect repeats, each forming a helix-turn-helix structure of about 53 amino acids. Three regularly spaced tryptophan residues, which form a tryptophan cluster in the three-dimensional helixturn-helix structure, are characteristic of a MYB repeat (Stracke et al. 2001).

MYB proteins can be classified into three subfamilies depending on the number of adjacent repeats in the MYB domain (one, two or three) (Jin and Martin 1999). We refer to MYB-like proteins with one repeat as 'MYB1R factors', with two as 'R2R3-type MYB' factors, and with three repeats as 'MYB3R' factors. In high plants, R2R3-MYB is the most common type (Stracke et al. 2001). Variation within certain conserved C-terminal motifs has been used to classify plant R2R3-MYB genes into 22 subgroups, with members within each subgroup predicted to share similar or identical functions (Kranz et al. 1998). Numerous R2R3MYB genes have been found to be involved in the control of plant specific processes, including primary and secondary metabolism, hormonal signaling, stress responses, cell fate and identity (Gocal et al. 2001; Liu et al. 2008; Payne et al. 1999; Schmitz et al. 2002; Tamagnone et al. 1998). A 
number of R2R3-MYB genes play important roles in the responses to abiotic stresses in plant. Arabidopsis R2R3MYB genes including AtMYB2, AtMYB15, AtMYB41, AtMYB70, and AtMYB73 were shown to be associated with abiotic stresses tolerance (Cheong et al. 2002; Cominelli et al. 2008; Ding et al. 2009; Fowler and Thomashow 2002; Urao et al. 1993). Other plant species R2R3-MYB genes involved in abiotic stress tolerance have been reported (Qin et al. 2012; Shan et al. 2012).

Soybean (Glycine max) is the major source of edible vegetable oil, and the dominant source of high-quality protein for livestock and humans. Its growth and yield were affected by various abiotic stresses. Soybean R2R3-MYB genes such as GmMYB76, GmMYB92 and GmMYB177 are related to abiotic stresses tolerance (Liao et al. 2008). In the present study, the expressions of a soybean R2R3-MYB gene designated as GmMYB184 were analyzed in response to drought, salt, cold, and abscisic acid (ABA) treatments. We propose the possible role of GmMYB184 involved in abiotic stress tolerance in plant.

\section{Materials and methods}

Plant materials and stress treatments

Soybean (Glycine max L. Gwangan) was grown in a greenhouse. The 3-4 week-old soybean plants were treated with low temperature $\left(4^{\circ} \mathrm{C}\right)$, drought, or high salinity $(100 \mathrm{mM}$ $\mathrm{NaCl})$ stresses and $\mathrm{ABA}(100 \mu \mathrm{M})$ as previously described (Chung et al. 2009). Plant samples were immediately frozen in liquid nitrogen and stored at $-75^{\circ} \mathrm{C}$.
Molecular cloning of GmMYB184

To obtain the full-length cDNA of GmMYB184, PCR was performed using soybean cDNA with a forward primer and a reverse primer (Table 1). Total RNA was isolated by RNA extraction kit (Ambion) and cDNA synthesis was carried out using cDNA synthesis kit (Promega). PCR condition was set to denature for $60 \mathrm{sec}$ at $94^{\circ} \mathrm{C}$, annealing for $60 \mathrm{sec}$ at $55^{\circ} \mathrm{C}$, extension for $1 \mathrm{~min} 30 \mathrm{sec}$ at $72^{\circ} \mathrm{C}$ with 30 cycles. The resulting PCR product was cloned into the pGEMT Easy Vector (Promega) and the resulting clone was sequenced subsequently (Genotech).

Multiple sequence alignment and phylogenetic analysis

Alignments of protein sequences were performed by the CLUSTALW program (Higgins et al. 1996) with default parameters. Potential orthologous MYB-like protein amino acid sequences were collected based on blastp homology searches with the GmMYB184 sequence using the NCBI (http://blast.ncbi.nlm.nih.gov/Blast.cgi). The neighbor-joining tree was constructed to identify a gene cluster orthologous to GmMYB184 after re-adjustments of the sequence alignment with Jalview sequence editing program (http://www.jalview.org). Phylogenetic analysis was based on the neighbor-joining tree using the MAFFT web-based alignment program (http:// align.bmr.kyushu-u.ac.jp/mafft/online/server/index.html) with 1000 bootstrap replications.

RT-PCR and real-time (q)RT-PCR analysis

Total RNA was isolated using an RNA extraction kit (Ambion). The complementary DNA (cDNA) was synthesized from $2 \mu \mathrm{g}$ of total RNA using oligo d(T)18 primer, dNTP

Table 1 Primers used for (q)RT-PCR analyses and cloning

\begin{tabular}{lll}
\hline Gene & \multicolumn{1}{c}{ Forward primer (5'-3') } & \multicolumn{1}{c}{ Reverse primer (5'-3') } \\
\hline $\begin{array}{l}\text { (q)RT-PCR } \\
\text { GmMYB184 } \\
\text { TubulinA }\end{array}$ & F-GCTGCTTCAACTAGCCCAAC & R-GTCCCAAACGACGAAACTGT \\
\hline $\begin{array}{l}\text { Cloning } \\
\text { pGEMT-Easy }\end{array}$ & F-TTCTCCATTATTCAAACTGT & R-CACCAAAATAGAAGCATAAT \\
$\begin{array}{l}\text { GmMYB184 } \\
\text { pENTR }\end{array}$ & F-ATGTCTACTTCAAAGAGCGTCA & \\
$\begin{array}{l}\text { GmMYB184 } \\
\text { pGBK-T7 }\end{array}$ & F-CACCATGTCTACTTCAAAGAGC & R-TTATTTTTGTAACTTGCTAAATTGCC \\
GmMYB184 (F) & F-GAATTCATGTCTACTTCAAAGAGC & R- TTATTTTTGTAACTTGCTAAATTGCC \\
GmMYB184 (N) & F-GAATTCATGTCTACTTCAAAGAGC & R-GTCGACTTATTTTTGTAACTTGC \\
GmMYB184 (C) & F-GAATTCATGCCTAGATTGCTTCAG & R-GTCGACTCAGTTTCGCCTAACTTG \\
\hline
\end{tabular}


and M-MLV reverse transcriptase (Invitrogen). PCR amplification was performed using $1 \mu \mathrm{l}$ cDNA as a template for 26 cycles of: $94^{\circ} \mathrm{C}$ for $50 \mathrm{~s}, 55^{\circ} \mathrm{C}$ for $50 \mathrm{~s}$, and $72^{\circ} \mathrm{C}$ for $50 \mathrm{~s}$. Real-time (q)PCR was performed for 40 cycles using $1 \mu \mathrm{l}$ cDNA as a template and CFX-96TM RealTime system with SYBR Premix (Bio-Rad). (q)RT-PCR data were analyzed with CFX Manager v2.1 software (Bio-Rad). Data was normalized to Tubulin A mRNA levels. The primers used for RT-PCR analysis are listed in Table 1.

Generation of GFP fusion construct and subcellular localization

The full-length GmMYB184 cDNA was PCR-amplified with a CACC-forward primer and a reverse primer, respectively (Table 1). Each PCR amplified DNA fragment was cloned between attB1 and attB2 sites of the $\mathrm{pENTR/D} \mathrm{TOPO} \mathrm{vector}$ (Invitrogen, USA; http://www.invitrogen.com/). GmMYB184 was then recombined as a C-terminal fusion of GFP into the Gateway destination binary vector, pK7FGW2 (Plant Systems Biology, Belgium; http://www.psb.ugent.be/), yielding 35S:GFP-GmMYB184 by a LR recombination reaction.

Each 35S:GFP, or 35S:GFP-GmMYB184 construct was transformed into Agrobacterium sp. strain C58c1, respectively. For the transient expression of GFP proteins in planta, the transformed Agrobacterium cells containing 35S:GFP, or 35S:GFP-GmMYB184 were inoculated into the leaves of Nicotiana benthamiana plants as previously described (Chung et al. 2004). The epidermal tissues were peeled from the Agrobacterium-infiltrated $N$. benthamiana leaves to detect GFP image. A Zeiss LSM700 (Germany) confocal microscope was used to observe fluorescence as described previously (Chung et al. 2009).

\section{Yeast transactivation test}

For the bait vector construction, the full-length (1-312 aa), N-terminal (1-140 aa) and C-terminal (141-312 aa) region of GmMYB184 was PCR-amplified with a forward primer with EcoRI and a reverse primer with SalI (Table 1). PCR products were cleaved with EcoRI and SalI and were introduced into the pGBK-T7. The yeast strain AH109 (Clontech) was transformed with pGBK-T7 (DBD), pGBKGmMYB184-F (DBD-F), pGBK-GmMYB184-N (DBD-N), or pGBK-GmMYB184-C (DBD-C), respectively. Yeast cells were plated onto $\mathrm{SD} /$-Trp medium and incubated for $4 \mathrm{~d}$ at $30^{\circ} \mathrm{C}$. Transformants were subsequently grown on $\mathrm{SD} /$ -Trp/-His or YPAD medium for $3 \mathrm{~d}$ at $30^{\circ} \mathrm{C}$. Transcriptional activity of GmMYB184 was analyzed in yeast. To measure the transcriptional activation, $\beta$-galactosidase assays were carried out using O-nitrophenyl- $\beta$-D-galactopyranoside (ONPG) as a substrate following the manufacturer's instructions (Invitrogen).

\section{Results and discussion}

Soybean GmMYB184 encodes an R2R3-type MYB transcription factor

To understand the molecular mechanism during osmotic stress in soybean, molecular characterization of a soybean gene GmMYB184 (Genbank accession number; NM_001248908) encoding an R2R3-MYB TF was carried out in this study. Deduced amino acid sequences of GmMYB184 are homologous to an R2R3-type MYB transcription factor. GmMYB184 cDNA is composed of an open reading frame (ORF) of 939 nucleotides (nts) encoding $36.3 \mathrm{kDa}$ polypeptide with 312 aa residues and $\mathrm{pI}$ of 7.15 (Genbank number; JX090252) (Fig. 1A). GmMYB184 has the MYB domain that is composed of two imperfect repeat SANT sequences characteristic of R2 (16-69 aa) and R3 (71-133 aa) (Fig. 1A). NetNES1.1 predicted that R3 region of GmMYB184 contains a Leu-rich nuclear export signal (NES) (Fig. 1A). Multiple sequence alignment analysis showed that the $\mathrm{N}$-terminal region represents R2R3-type DNA binding domain highly conserved among GmMYB184, GmMYB84 and MYBs from other plants (Fig. 1A). Sequence identities of GmMYB184 with MYBs from other plants have wide range such as VvMYB (CBI15464) 75\%, PtMYB (EEE96766) 56\%, CsMYB (ADF43752) 56\%, MtMYB (AES62892) 47\%, AtMYB116 (NP 564230) 44\% (Fig. 1A). In soybean genome, GmMYB84 shares 92\% identity with GmMYB184, indicating that GmMYB84 and GmMYB184 are homologous (Fig. 1A).

Phylogenetic analysis using the deduced amino acid sequences of GmMYB184 and sixteen plant R2R3-MYB proteins was inferred on the basis of the multiple sequence alignment (Fig. 1B). Phylogenetic relationship between soybean GmMYB184 and similar R2R3-MYB proteins shows that GmMYB184 protein shares higher similarity to MYBs from monocotyledons (58-74\%) than MYB (47\%) of leguminous Medicago truncatula or Arabidopsis AtMYB116 and AtMYB62 (45\%) (Fig. 1B). The phylogenetic tree shows that GmMYB184 are clustered with GmMYB84 and but diverged from GmMYB6-like protein (Fig. 1B). GmMYB184 shares limited identities with soybean MYB transcription factors deposited in NCBI database, of which GmMYB6-like protein (XP_003534166) ranked as the top with high identity 


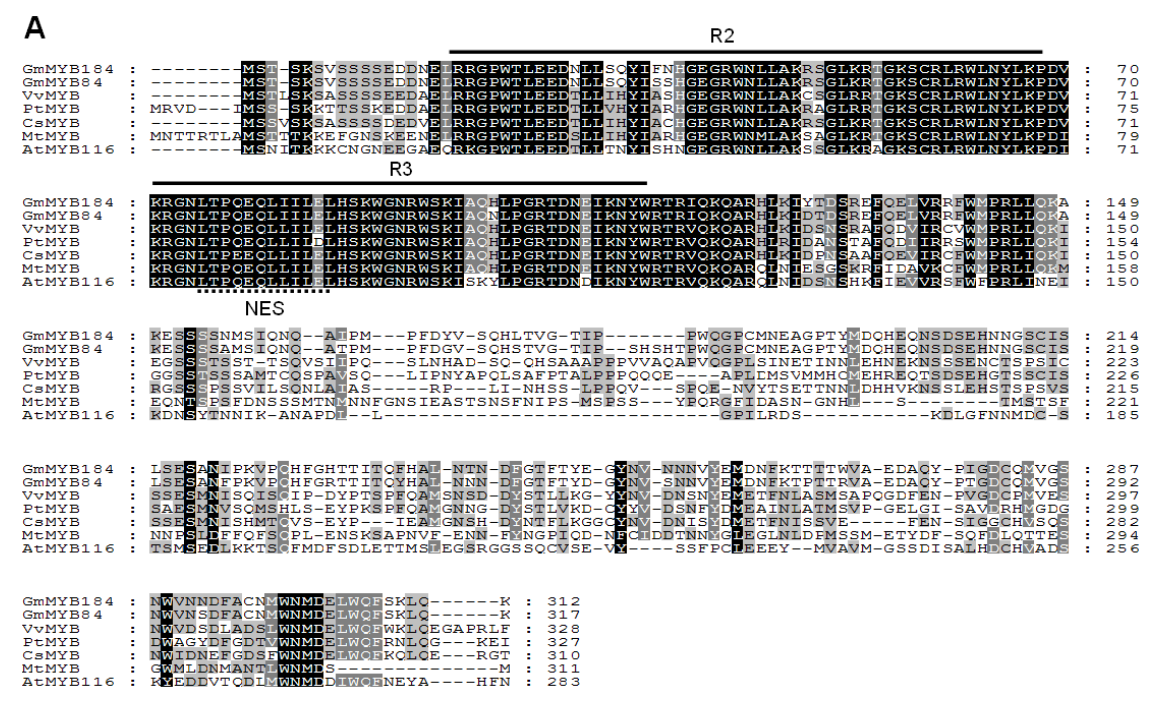

B

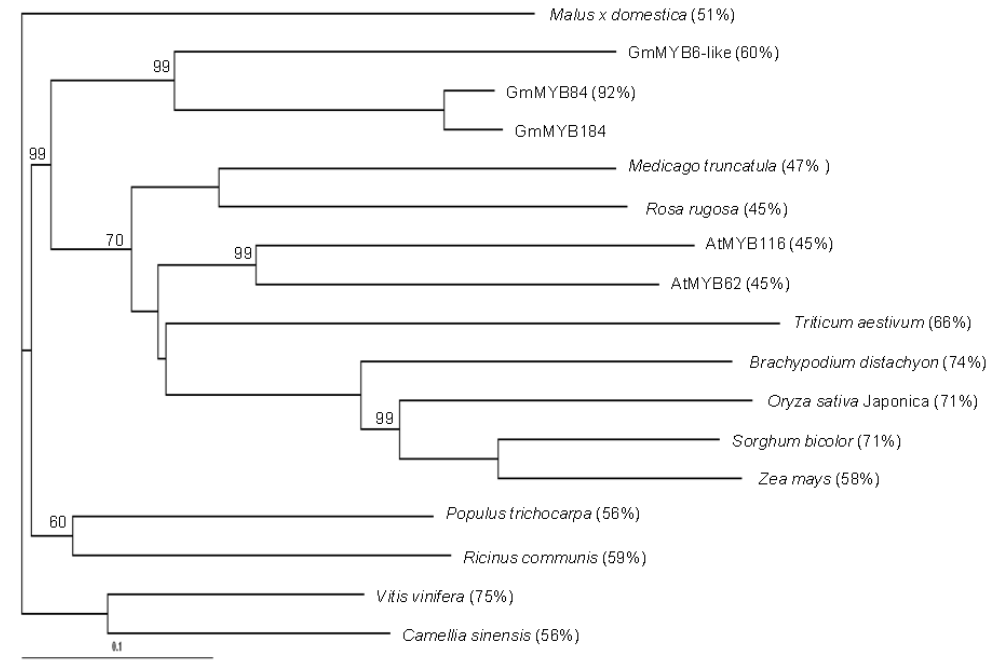

Fig. 1 Alignment and phylogenetic relationship of GmMYB184 with other R2R3-MYB like proteins. (A) A multiple sequence alignment of deduced polypeptide sequence of GmMYB184 with other R2R3-MYB like protein sequences; GmMYB84 (G. max, ABH02839); VvMYB (Vitis vinifera, CBI15464), PtMYB (Populus trichocarpa, EEE96766), CsMYB (Camellia sinensis, ADF43752), MtMYB (Medicago truncatula, AES62892), AtMYB116 (A. thaliana, NP_564230). The N-terminal conserved DBD domain is depicted as an overline showing $\mathrm{R} 2$ and R3. The nucleus export signal (NES) is indicated by the dotted underline. (B) The numbers on each line indicate bootstrap values. R2R3-MYB like proteins similar with GmMYB184 were retrieved from databases including A. thaliana (NP_564230, At1G25340; NP_176999, At1G68320), Oryza sativa Japonica (BAB18296), P. trichocarpa (EEE96766), G. max (GmMYB84, ABH02839; GmMYB6-like protein, XP_003534166) M. truncatula (AES62892), Zea mays (ACF85620), V. vinifera (CBI15464), C. sinensis (ADF43752), Malux $x$ domestica (ABB84756), Ricinus communis (EEF27793), Rosa rugosa (ADG56766), Sorghum bicolor (BAB18296), Brachypodium distachyon (XP_003565287) and Triticum aestivum (ABU93236). To evaluate the statistical support for tree topology, bootstrap values were calculated using from 100 to 1000 replicates

$(60 \%)$, indicating that $G m M Y B 184$ is a new member of MYB genes in soybean.

RNA expression of the GmMYB184 gene under osmotic stress conditions

RNA expression of GmMYB184 was investigated in response to various stress treatments (Fig. 2). RNA expression of GmMYB184 gene was specifically induced at $6 \mathrm{~h}$ and significantly up-regulated at $24 \mathrm{~h}$ after $\mathrm{ABA}$ application (Fig. 2). ABA is closely related to osmotic stresses such as drought, low temperature and salinity in plant (Himmelbach et al. 2003). We examined GmMYB184 expression by other abiotic stresses such as drought, low temperature, and salt stress (Fig. 2). GmMYB184 was significantly induced by drought stress, but weakly by the low temperature (Fig. 2). High salinity stress significantly induced GmMYB184 expression after the long time exposure (24 h) (Fig. 2). This 
shows that soybean GmMYB184 RNA expression is induced by diverse osmotic stresses such as drought, low temperature and high salinity stress, implying that GmMYB184 is involved in osmotic stress response in plant.

Plant MYBs are implicated in numerous processes including responses to environmental stress (Dubos et al. 2010; He et al. 2012; Stracke et al. 2001; Zhang et al. 2012). MYBs have been found to be involved in ABAdependent or ABA-independent signaling pathways. The AtMYB2 (Urao et al. 1993), CpMYB10 (Villalobos et al.

A
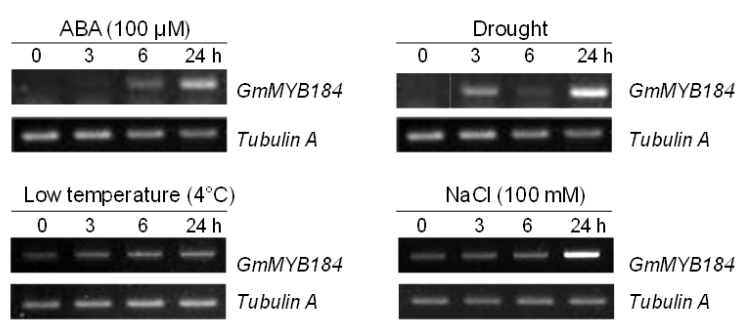

B
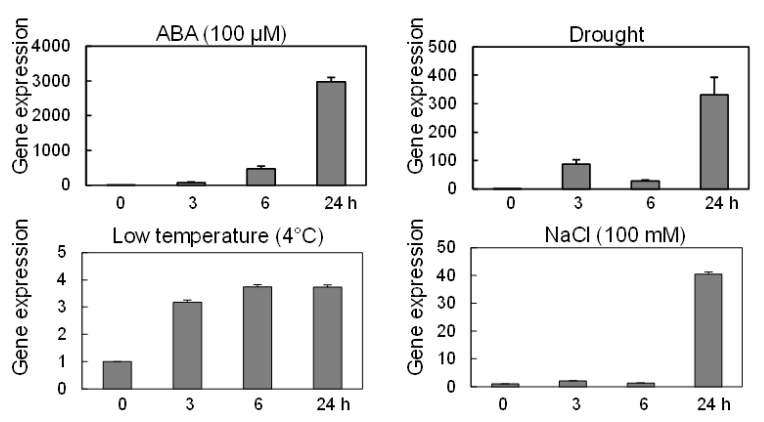

Fig. 2 RNA expression of GmMYB184 under abiotic stress conditions using RT-PCR and (q)RT-PCR analyses. Soybean plants were sprayed with ABA $(100 \mu \mathrm{M})$, or subjected to drought, low temperature $\left(4^{\circ} \mathrm{C}\right)$, or high salinity stresses $(100 \mathrm{mM} \mathrm{NaCl})$. Transcript levels of GmMYB184 were quantified by (q)RT-PCR against tubulin transcript level. Each value is the mean $\pm \mathrm{SD}$ of three independent biological determinations
2004), and HOS10 (Zhu et al. 2005) genes are induced by $\mathrm{ABA}$, and also induced by drought, salt, chilling, or heat treatments. However, the genes HPPBF-1 (Nagaoka and Takano 2003), Osmyb4 (Vannini et al. 2004), and AtMYB61 (Liang et al. 2005) are not induced by ABA, but can enhance drought, salt, or freezing tolerances. The soybean GmMYB76, GmMYB92, and GmMYB177 genes were shown to be induced by various abiotic stresses but not to be significantly affected by ABA treatment (Liao et al. 2008). In the present study, GmMYB184 was specifically responsive to ABA during late time (Fig. 2).

GmMYB184 localizes in the nuclei and has transcriptional activity in yeast

GmMYB184 protein was predicted to be located in the nucleus based on the prediction by Nuc-PLoc (http://www. csbio.sjtu.edu.cn/bioinf/Nuc-PLoc/) (Shen and Chou 2007). To confirm this prediction, we transiently expressed GFPGmMYB184 fusion protein in the $N$. benthamiana leaves (Fig. 3). As shown in Fig. 3, the control GFP was distributed uniformly in the whole cell, whereas the GFP-GmMYB184 fusion protein was targeted to the nucleus (Fig. 3).

The transactivation activities of GmMYB184 were analyzed using a yeast assay system (Fig. 4). GAL4 DNA binding domain (DBD)-GmMYB184 fusion plasmids (F, full-length; $\mathrm{N}, \mathrm{N}$-terminal region; $\mathrm{C}, \mathrm{C}$-terminal region) were transformed into yeast cells and assayed for their ability to activate transcription of the marker HIS3 gene controlled by the GAL4 upstream activation sequence and their ability to promote yeast growth in the absence of histidine (Fig. 4A). The $\beta$-galactosidase activity was also examined for the marker LacZ gene expression (Fig. 4B). Figure 4 showed that either its full-length region or the

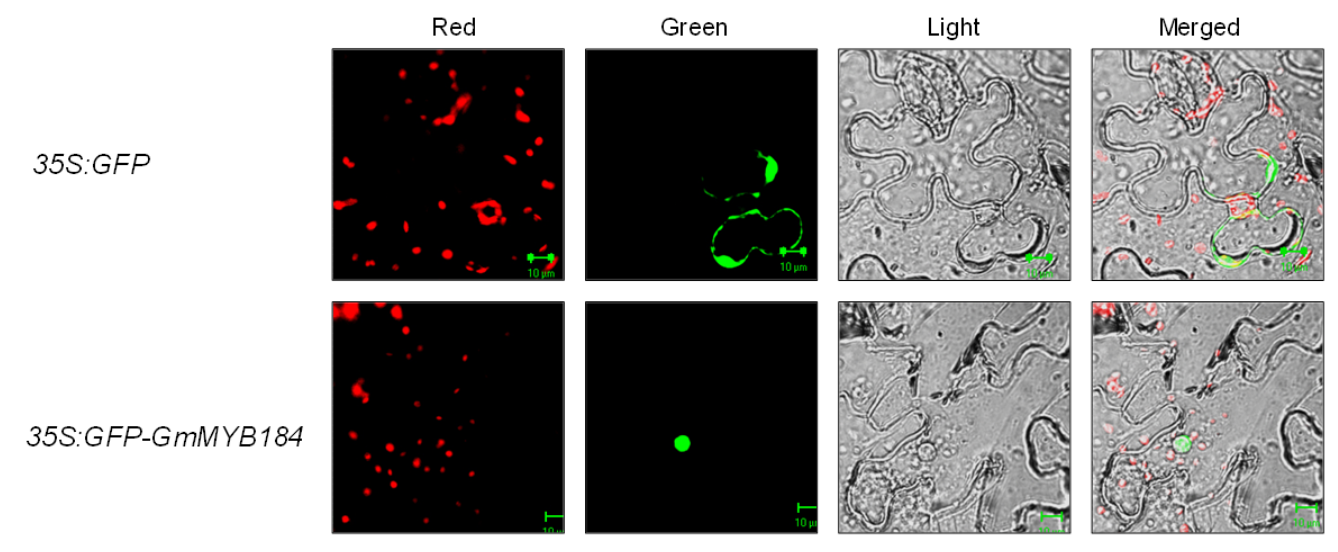

Fig. 3 Subcellular localization of GFP and GFP-GmMYB184 in tobacco protoplast. (A) GFP or GFP-GmMYB184 was transiently expressed in Nicotiana benthamiana using Agrobacterium-mediated transformation method. The epidermis was observed with a Leica SP2-AOBS confocal microscopy. The scale bar represents $10 \mu \mathrm{m}$. For each panel, Light shows the actual image, red channel shows chloroplast fluorescence and green channel shows GFP fluorescence 
A
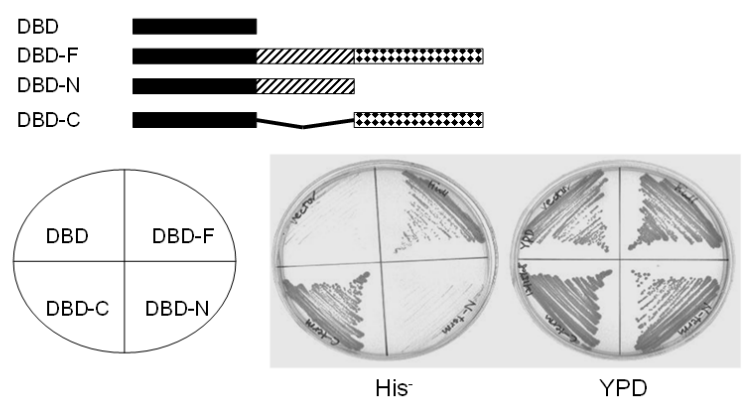

B

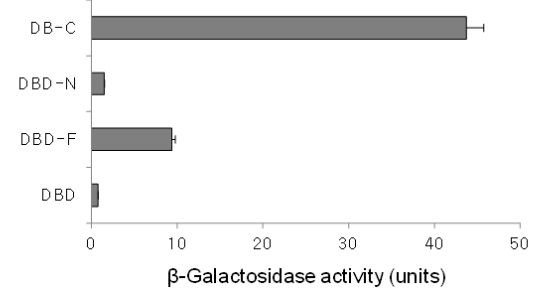

Fig. 4 Transcriptional activation analysis of GmMYB184. (A) GAL4 DBD was fused to the full-length $(\mathrm{F}), \mathrm{N}$-terminal $(\mathrm{N})$ or C-terminal (C) region of GmMYB184. Growth of the transformants on $\mathrm{SD} /$-His and YPAD media is shown to the transactivation activity. (B) AH109 yeasts transformed with pGBK-T7 (DBD), pGBK-GmMYB184-F (DBD-F), pGBK-GmMYB184-N (DBD-N) or pGBK-GmMYB184-C (DBD-C) were tested for $\beta$-gal activities sing ONPG assays. The $\beta$-gal activities were measured with 3 replications

C-terminal region of GmMYB184 had transactivation capacity indicating that the C-terminal region is essential for the activation of the transcription of reporter genes LacZ and His in the genome of the yeast AH109 strain. Consistently, $\beta$-galactosidase activity analysis indicated that the $\beta$-galactosidase units of the transformant with DBD-F or $-\mathrm{C}$ were much higher than that of DBD-N, and those of DBD-C were also significantly higher than those of DBD-F (Fig. 4B), demonstrating that the N-terminal region of GmMYB might have the repression effect for transactivation activities in yeast. Yeast transactivaion assays indicated that GmMYB184 has transactivation activity, which is attributed to its $\mathrm{C}$-terminal region.

Most MYB proteins are presumed to be transcriptional activators with $\mathrm{ADs}$ in the $\mathrm{C}$-terminal region similar to the acidic AD C-terminal region found in $c$-MYB (Weston 1998). Compared to the highly conserved N-terminal DBD, the C-terminal regions of MYB proteins are fairly flexible (Fig. 2A) (Kranz et al. 1998). In some cases, the C-terminal domains of MYBs may show transactivation repression effects or interaction activities with other transcription factors (Martin and Paz-ares 1997). In this study, we show that GmMYB184 has transactivation activities and the C-terminal region is essential for transactivation ability in yeast assay system.
MYB genes are a super-family of transcription factors, some of which are found to be involved in abiotic stress response. In this study, we have cloned and characterized GmMYB184 encoding R2R3-type MYB TF from soybean. Inducible expression of GmMYB184 under osmotic stress and $A B A$ treatment implies its involvement in osmotic stress tolerance in ABA-dependent manner. We have also confirmed that it is targeted to the nucleus in plant and its C-terminal region is essential for the transactivation activity in yeast. In conclusion, we propose that GmMYB184 encoding R2R3-type MYB TF is involved in molecular events in osmotic stress and ABA.

\section{Acknowledgements}

This research was supported by Basic Science Research Program through the National Research Foundation of Korea (KRF) funded by the Ministry of Education, Science and Technology (KRF-2012-001205 and -2012-001273), and the Next-Generation BioGreen 21 Program (Plant Molecular Breeding Center No. PJ007970), Rural Development Administration, Republic of Korea.

\section{References}

Cheong YH, Chang HS, Gupta R, Wang X, Zhu T, Luan S (2002) Transcriptional profiling reveals novel interactions between wounding, pathogen, abiotic stress, and hormonal responses in Arabidopsis. Plant Physiol 129:661-677

Chung E, Seong E, Kim Y-C, Chung EJ, Oh S-K, Lee S, Park JM, Joung YH, Choi D (2004) A method of high frequency virus-induced gene silencing in chili pepper (Capsicum annum L. cv. Bukang). Mol Cells 17:377-380

Chung E, Cho CW, Yun BH, Choi HK, So HA, Lee SW, Lee J-H (2009) Molecular cloning and characterization of the soybean DEAD-box RNA helicase gene induced by low temperature stress. Gene 443:91-99

Cominelli E, Sala T, Calvi D, Gusmaroli G, Tonelli C (2008) Overexpression of the Arabidopsis AtMYB41 gene alters cell expansion and leaf surface permeability. Plant J 53:53-64

Ding ZH, Li SM, An XL, Liu X, Qin HJ, Wang DW (2009) Transgenic expression of MYB15 confers enhanced sensitivity to abscisic acid and improved drought tolerance in Arabidopsis thaliana. J Genet Genomics 36:17-29

Dubos C, Stracke R, Grotewold E, Weisshaar B, Martin C, Lepiniec L (2010) MYB transcription factors in Arabidopsis. Trends Plant Sci 15:573-581

Fowler S, Thomashow MF (2002) Arabidopsis transcriptome profiling indicates that multiple regulatory pathways are activated during cold acclimation in addition to the $\mathrm{CBF}$ cold 
response pathway. Plant Cell 14:1675-1690

Gocal GFW, Sheldon CC, Gubler F, Moritz T, Bagnall DJ, MacMillan CP, Li SF, Parish RW, Dennis ES, Weigel D, King RW (2001) GAMYB-like genes, flowering, and gibberellin signaling in Arabidopsis. Plant Physiol 127:1682-1693

He Y, Li W, Lv J, Jia Y, Wang M, Xia G (2012) Ectopic expression of a wheat MYB transcription factor gene, TaMYB73, improves salinity stress tolerance in Arabidopsis thaliana. J Exp Bot 63:1511-1522

Higgins DG, Thompson JD, Gibson TJ (1996) Using CLUSTAL for multiple sequence alignments. Methods Enzymol 266: 383-402

Himmelbach A, Yang Y, Grill E (2003) Relay and control of abscisic acid signaling. Curr Opin Plant Biol 6:470-479

Jin H, Martin C (1999) Multifunctionality and diversity within the plant MYB-gene family. Plant Mol Biol 41:577-585

Klempnauer KH, Gonda TJ, Bishop JM (1982) Nucleotide sequence of the retroviral leukemia gene $\mathrm{v}$-myb and its cellular progenitor c-myb: the architecture of a transduced oncogene. Cell 31:453-463

Kranz HD, Denekamp M, Greco R, Jin H, Leyva A, Meissner RC, Petroni K, Urzainqui A, Bevan M, Martin C, Smeekens S, Tonelli C, Paz-Ares J, Weisshaar B (1998) Towards functional characterization of the numbers of the R2R3-MYB gene family from Arabidopsis thaliana. Plant J 16:263-276

Liang YK, Dubos C, Dodd IC, Holroyd GH, Hetherington AM, Campbell MM(2005) AtMYB61, an R2R3-MYB transcription factor controlling stomatal aperture in Arabidopsis thaliana. Curr Biol 15:1201-1206

Liao Y, Zou HF, Wang HW, Zhang WK, Ma B, Zhang JS, Chen SY (2008) Soybean GmMYB76, GmMYB92, and GmMYB177 genes confer stress tolerance in transgenic Arabidopsis plants. Cell Res 18:1047-1060

Lipsick JS (1996) One billion years of Myb. Oncogene 13: 223-235

Liu L, Du H, Tang XF, Wu YM, Huang YB, Tang YX (2008) The roles of MYB transcription factors on plant defense responses and its molecular mechanism. Hereditas 30:1265-1271

Martin C, Paz-ares J (1997) MYB transcription factors in plants. Trends Genet 13:67-73

Nagaoka S, Takano T (2003) Salt tolerance-related protein STO binds to a Myb transcription factor homologue and confers salt tolerance in Arabidopsis. J Exp Bot 54:2231-2237

Nakashima K, Ito Y, Yamaguchi-Shinozaki K (2009) Transcriptional regulatory networks in response to abiotic stresses in Arabidopsis and grasses. Plant Physiol 149:88-95

Payne T, Clement J, Arnold D, Lloyd A (1999) Heterologous myb genes distinct from GL1 enhance trichome production when overexpressed in Nicotiana tabacum. Development 126: 671-682

Qin Y, Wang M, Tian Y, He W, Han L, Xia G (2012) Overexpression of TaMYB33 encoding a novel wheat MYB transcription factor increases salt and drought tolerance in
Arabidopsis. Mol Biol Rep 39:7183-7192

Schmitz G, Tillmann E, Carriero F, Fiore C, Theres K (2002) The tomato Blind gene encodes a MYB transcription factor that controls the formation of lateral meristems. Proc Natl Acad Sci USA 99:1064-1069

Schwechheimer C, Zourelidou M, Bevan MW (1998) Plant transcription factor studies. Ann Rev Plant Physiol Plant Mol Biol 49:127-150

Shan H, Chen S, Jiang J, Chen F, Chen Y, Gu C, Li P, Song A, Zhu X, Gao H, Zhou G, Li T, Yang X (2012) Heterologous expression of the Chrysanthemum R2R3-MYB transcription factor CmMYB2 enhances drought and salinity tolerance, increases hypersensitivity to ABA and delays flowering in Arabidopsis thaliana. Mol Biotechnol 51:160-173

Shen H-B, Chou K-C (2007) "Nuc-PLoc: A new web-server for predicting protein subnuclear localization by fusing PseAA composition and PsePSSM'. Protein Eng Des Sel 20:561-567

Shinozaki K, Yamaguchi-Shinozaki K (2007) Gene networks involved in drought stress response and tolerance. J Exp Bot 58:221-227

Singh K, Foley RC, Onate-Sanchez L (2002) Transcription factors in plant defense and stress responses. Curr Opin Plant Biol 5: 430-436

Stracke R, Werber M, Weisshaar B (2001) The R2R3-MYB gene family in Arabidopsis thaliana. Curr Opin Plant Biol 4: 447-456

Tamagnone L, Merida A, Parr A, Mackay S, Culianez-Macia FA, Roberts K, Martin C (1998) The AmMYB308 and AmMYB330 transcription factors from antirrhinum regulate phenylpropanoid and lignin biosynthesis in transgenic tobacco. Plant Cell 10: 135-154

Urao T, Yamaguchi-Shinozaki K, Urao S, Shinozaki K(1993) An Arabidopsis myb homolog is induced by dehydration stress and its gene product binds to the conserved MYB recognition sequence. Plant Cell 5:1529-1539

Vannini C, Locatelli F, Bracale M, Magnani E, Marsoni M, Osnato M, Mattana M, Baldoni E, Coraggio I (2004) Overexpression of the rice Osmyb4 gene increases chilling and freezing tolerance of Arabidopsis thaliana plants. Plant J 37:115-127

Villalobos MA, Bartels D, Iturriaga G (2004) Stress tolerance and glucose insensitive phenotyes in Arabidopsis overexpressing the CpMYB10 transcription factor gene. Plant Physiol 135: 309-324

Weston K (1998) Myb proteins in life, death and differentiation. Curr Opin Genet Dev 8:76-81

Zhang L, Zhao G, Jia J, Liu X, Kong X (2012) Molecular characterization of 60 isolated wheat MYB genes and analysis of their expression during abiotic stress. J Exp Bot 63:203-214

Zhu JH, Verslues PE, Zheng XW, Lee BH, Zhan X, Manabe Y, Sokolchik I, Zhu Y, Dong CH, Zhu JK, Hasegawa PM, Bressan RA (2005) HOS10 encodes an R2R3-type MYB transcription factor essential for cold acclimation in plants. Proc Natl Acad Sci USA 102:9966-9971 